\title{
Degree-Based Topological Properties of Molecular Polymeric Networks Composed by Sierpinski Networks
}

\author{
Abdul Rauf $\mathbb{D}^{1},{ }^{1}$ Muhammad Ishtiaq $\mathbb{D}^{1},{ }^{1}$ Hafiz Faraz Qaiser, ${ }^{1}$ Adnan Aslam $\mathbb{D}^{2}$, \\ and Kraidi Anoh Yannick $\mathbb{D}^{3}$ \\ ${ }^{1}$ Department of Computer Science and Mathematics, Air University Multan Campus, Multan, Pakistan
${ }^{2}$ Department of Natural Sciences and Humanities, University of Engineering and Technology, Lahore (RCET), Pakistan
${ }^{3}$ UFR of Mathematics and Computer Science, University Felix Houphouet Boigny of Cocody, Abidjan, Côte d'Ivoire
}

Correspondence should be addressed to Kraidi Anoh Yannick; kayanoh2000@yahoo.fr

Received 5 August 2021; Accepted 15 September 2021; Published 8 October 2021

Academic Editor: Tabasam Rashid

Copyright (c) 2021 Abdul Rauf et al. This is an open access article distributed under the Creative Commons Attribution License, which permits unrestricted use, distribution, and reproduction in any medium, provided the original work is properly cited.

Sierpinski graphs are a widely observed family of fractal-type graphs relevant to topology, Hanoi Tower mathematics, computer engineering, and around. Chemical implementations of graph theory establish significant properties, such as chemical activity, physicochemical properties, thermodynamic properties, and pharmacological activities of a molecular graph. Specific graph descriptors alluded to as topological indices are helpful to predict these properties. These graph descriptors have played a key role in quantitative structure-property/structure-activity relationships (QSPR/QSAR) research. The objective of this article is to compute Randic index $\left(R_{-1 / 2}\right)$, Zagreb index $\left(M_{1}\right)$, sum-connectivity index (SCI), geometric-arithmetic index (GA), and atombond connectivity $(\mathrm{ABC})$ index based on ev-degree and ve-degree for the Sierpinski networks $S(n, m)$.

\section{Introduction}

Graph theory is concerned with network of points connected by lines. The beginning of graph theory deals with recreational math problems, but now it has grown into significant research area. Recently, it acquires sufficient attention due to its use in computer science, circuit boards, interrelated systems, living systems, sociology, and so on. Chemical graph theory (CGT) plays a very vital role in the composition and arrangement of substances. Chemical compounds are usually supposed to be the chemical networks, consequently whose compound's atoms represent vertices, and bonds represent edges of a graph. Scientists are very attentive to know about the topology of chemical networks through some mathematical parameters gained from the network graphs of chemical molecules. A large scale of topological indices has been examined in biochemistry through QSPR/QSAR analysis to analyze chemical networks topology related to pharmacy, medical engineering, and experimental science, which are important fields of CGT [1]. A quick study of QSPR/QSAR is one of the main reasons to apply graph theory in chemistry $[2,3]$.
The required technique for the study of some graphical structures is to break down the structures into substructures containing significant properties and also some associated structures that are supposed to have similar properties. We need to know how the network-related substructures are similar to each other in these circumstances. For example, to make polymeric networks, Sierpinski graphs are used. A class of polymeric materials which are directly or indirectly linked together as polymeric chains are polymeric networks. In mathematics, fractal theory and other areas of science, the Sierpinski, and these types of different graphs are used. In the recent decade, fractal nature-based graphs such as Sierpinski and related graphs were studied with applications in topology and computer science. Nowadays, the modern communication systems like radio and mobile radiations as far as pattern-based log-periodic behaviour have been studied by fractal nature of Sierpinski networks. The family of Sierpinski graphs has some unique properties and was discussed in the literature $[4,5]$.

Topological indices are numerical numbers associated with the networks that can be helpful to predict some of its 
properties. Topological indices are mostly used for QSPR and QSAR studies. QSAR/QSPR studies have a lot of use in biochemistry and modern chemistry. It is important to select an appropriate descriptor to get a good correlation. There are many descriptors that can predict the simple properties of a molecular structure and give an insight into the properties of the structure under consideration [6]. Hence, we need to employ such molecular descriptors that can help to characterize the molecular properties and influence its activities [4, 7-9]. Harold Wiener introduces the concept of path number of a chemical graph and later calls it Wiener index. After that many topological indices were introduced this theory became the central topic of research. The most studied topological indices among these are the degreebased having many applications in chemical graph theory.

Let $\mathscr{H}$ be a simple connected graph with its edge set and vertex set denoted by $E$ and $V$, respectively. The order of $\mathscr{H}$ is the cardinality of set $V$. Similarly, the size of $\mathscr{H}$ is the cardinality of set $E$. Let $u_{1} \in V$, then $N\left(u_{1}\right)$ denotes the set containing neighbors of $u_{1}$ and is called open neighborhood of $u_{1}$. The closed neighborhood of a vertex $u_{1}$ is denoted by $N\left[u_{1}\right]$ and defined as $N\left[u_{1}\right]=\left\{u_{1}\right\} \cup N\left(u_{1}\right)$. We denote the degree of a vertex $u_{1}$ by $\Lambda\left(u_{1}\right)$ and count of elements in $N\left(u_{1}\right)$. For basic concepts related to graph theory, we refer the readers to the book by West [10].

First degree-based topological index was proposed by Randic [5] in 1975 and named as "branching index." It was later called as Randic connectivity index $R_{(-1) / 2}(\mathscr{H})$. It was found to be useful to determine the degree of branching of saturated hydrocarbons in their carbon-atom skeleton. This index was later generalized by Bollobás and Erdos [11] in 1988 and they called it general Randic index. It was observed by Randic that a good correlation exists between various physical/chemical properties of alkanes and the Randic index $R_{\alpha}$. The mathematical formulas for Randić index and general Randić index are

$$
\begin{aligned}
R_{-(1 / 2)}(\mathscr{H}) & =\sum_{u_{1} v_{1} \in E(\mathscr{H})} \frac{1}{\sqrt{\Lambda\left(u_{1}\right) \Lambda\left(v_{1}\right)}}, \\
R_{\alpha}(\mathscr{H}) & =\sum_{u_{1} v_{1} \in E(\mathscr{H})}\left(\Lambda\left(u_{1}\right) \Lambda\left(v_{1}\right)\right)^{\alpha} .
\end{aligned}
$$

Gutman introduces the first and second Zagreb indices in [12]. These indices were first applied to the branching problem [13]. The mathematical formulas for Zagreb indices are

$$
\begin{aligned}
& M_{1}(\mathscr{H})=\sum_{u_{1} v_{1} \in E(\mathscr{H})} \Lambda\left(u_{1}\right)+\Lambda\left(v_{1}\right), \\
& M_{2}(\mathscr{H})=\sum_{u_{1} v_{1} \in E(\mathscr{H})}\left(\Lambda\left(u_{1}\right) \times \Lambda\left(v_{1}\right)\right) .
\end{aligned}
$$

The sum-connectivity index, denoted by SCI, was proposed by Zhou et al. [14] and has mathematical formula as

$$
\operatorname{SCI}(\mathscr{H})=\sum_{u_{1} v_{1} \in E(\mathscr{H})} \frac{1}{\sqrt{\Lambda\left(u_{1}\right)+\Lambda\left(v_{1}\right)}} .
$$

Zhong [15] introduced the harmonic index as follows:

$$
H(\mathscr{H})=\sum_{u_{1} v_{1} \in E(\mathscr{H})}\left(\frac{2}{\Lambda\left(u_{1}\right)+\Lambda\left(v_{1}\right)}\right) .
$$

Estrada et al. [16] introduced the atom-bond connectivity $(\mathrm{ABC})$ index which is formulated as

$$
\operatorname{ABC}(\mathscr{H})=\sum_{u_{1} v_{1} \in E(\mathscr{H})} \sqrt{\frac{\Lambda\left(u_{1}\right)+\Lambda\left(v_{1}\right)-2}{\Lambda\left(u_{1}\right) \Lambda\left(v_{1}\right)}} .
$$

This index gives a good stability model for cycloalkanes and linear and branched alkanes strain energy $[16,17]$.

Geometric-arithmetic index (GA) was put forward by Vukičević et al. in [18] and is defined as

$$
\mathrm{GA}(\mathscr{H})=\sum_{u_{1} v_{1} \in E(\mathscr{H})} \frac{2 \sqrt{\Lambda\left(u_{1}\right) \Lambda\left(v_{1}\right)}}{\Lambda\left(u_{1}\right)+\Lambda\left(v_{1}\right)} .
$$

Using octane isomers, it was proved that GA index shows good correlation with different physical/chemical properties.

Recently, two new degree definitions, namely, ve-degree and ev-degree, were proposed by Chellali et al. [19]. Their mathematical properties were discussed in more detail later by Horoldagva et al. [20]. We denote the ev-degree of an edge $u_{1} v_{1}=e_{1} \in E(\mathscr{H})$ by $\Lambda_{\mathrm{ev}}\left(e_{1}\right)$ and is defined as the cardinality of the set $N\left[u_{1}\right] \cup N\left[v_{1}\right]$. Similarly, for any vertex $v_{1}$, its ve-degree is denoted by $\Lambda_{\mathrm{ve}}\left(v_{1}\right)$ and is equal to the total number of edges that are incident to the vertices in the set $N\left[v_{1}\right]$. The classical degree-based topological indices definitions were converted into ev- and ve-degree. The Zagreb $\left(\mathrm{M}^{\mathrm{ev}}\right)$ index and Randic $\left(\mathrm{R}^{\mathrm{ev}}\right)$ index based on evdegree for any edge $e_{1}=u_{1} v_{1} \in E(\mathscr{H})$ are defined as

$$
\begin{aligned}
& \mathrm{M}^{\mathrm{ev}}(\mathscr{H})=\sum_{e_{1} \in E(\mathscr{C})} \Lambda_{\mathrm{ev}}\left(e_{1}\right)^{2}, \\
& \mathrm{R}^{\mathrm{ev}}(\mathscr{H})=\sum_{e_{1} \in E(\mathscr{H})} \Lambda_{\mathrm{ev}}\left(e_{1}\right)^{-(1 / 2)} .
\end{aligned}
$$

The first Zagreb alpha $\left(\mathrm{M}_{1}^{\alpha v e}\right)$ index of a vertex $v_{1} \in V(\mathscr{H})$ based on ve-degree is defined as

$$
\mathrm{M}_{1}^{\alpha \mathrm{ve}}(\mathscr{H})=\sum_{v_{1} \in V(\mathscr{H})} \Lambda_{\mathrm{ve}}\left(v_{1}\right)^{2} .
$$

Similarly, the ve-degree type first Zagreb beta $\left(\mathrm{M}_{1}^{\beta \mathrm{ve}}\right)$ index, geometric-arithmetic $\left(\mathrm{GA}^{\mathrm{ve}}\right)$ index, atom-bond connectivity $\left(\mathrm{ABC}^{\mathrm{ve}}\right)$ index, second Zagreb $\left(\mathrm{M}_{2}^{\mathrm{ve}}\right)$ index, sumconnectivity $\left(\chi^{\mathrm{ve}}\right)$ index, Randic $\left(\mathrm{R}^{\mathrm{ve}}\right)$ index, and harmonic $\left(\mathrm{H}^{\mathrm{ve}}\right)$ index for each edge $u_{1} v_{1} \in E(\mathscr{H})$ are defined as 


$$
\begin{aligned}
\mathrm{M}_{1}^{\beta \mathrm{ve}}(\mathscr{H}) & =\sum_{u_{1} v_{1} \in E(\mathscr{C})}\left(\Lambda_{\mathrm{ve}}\left(u_{1}\right)+\Lambda_{\mathrm{ve}}\left(v_{1}\right)\right), \\
\mathrm{M}_{2}^{\mathrm{ve}}(\mathscr{H}) & =\sum_{u_{1} v_{1} \in E(\mathscr{H})}\left(\Lambda_{v e}\left(u_{1}\right) \times \Lambda_{v e}\left(v_{1}\right)\right), \\
\operatorname{ABC}^{\mathrm{ve}}(\mathscr{H}) & =\sum_{u_{1} v_{1} \in E(\mathscr{H})} \sqrt{\frac{\Lambda_{\mathrm{ve}}\left(u_{1}\right)+\Lambda_{\mathrm{ve}}\left(v_{1}\right)-2}{\Lambda_{\mathrm{ve}}\left(u_{1}\right) \times \Lambda_{\mathrm{ve}}\left(v_{1}\right)}} \\
\mathrm{GA}^{\mathrm{ve}}(\mathscr{H}) & =\sum_{u_{1} v_{1} \in E(\mathscr{H})} \frac{2 \sqrt{\Lambda_{\mathrm{ve}}\left(u_{1}\right) \times \Lambda_{\mathrm{ve}}\left(v_{1}\right)}}{\Lambda_{\mathrm{ve}}\left(u_{1}\right)+\Lambda_{\mathrm{ve}}\left(v_{1}\right)}, \\
\mathrm{H}^{\mathrm{ve}}(\mathscr{H}) & =\sum_{u_{1} v_{1} \in E(\mathscr{H})} \frac{2}{\Lambda_{\mathrm{ve}}\left(u_{1}\right)+\Lambda_{\mathrm{ve}}\left(v_{1}\right)}, \\
\chi^{\mathrm{ve}}(\mathscr{H}) & =\sum_{u_{1} v_{1} \in E(\mathscr{H})}\left(\Lambda_{\mathrm{ve}}\left(u_{1}\right)+\Lambda_{\mathrm{ve}}\left(v_{1}\right)\right)^{-(1 / 2)}, \\
\mathrm{R}^{\mathrm{ve}}(\mathscr{H}) & =\sum_{u_{1} v_{1} \in E(\mathscr{H})}\left(\Lambda_{\mathrm{ve}}\left(u_{1}\right) \times \Lambda_{\mathrm{ve}}\left(v_{1}\right)\right)^{-(1 / 2)} .
\end{aligned}
$$

A lot of research is going on these topological indices. The results related to these topological indices can be found in [21-25]. The objective of this paper is to calculate exact values of topological indices for Sierpinski network $S(n, m)$ based on ve- and ev-degree.

\section{Methodology}

For the calculation and computation of topological indexes such as $\mathrm{M}^{\mathrm{ev}}, \mathrm{R}^{\mathrm{ev}}, \mathrm{M}_{1}^{\alpha \mathrm{ve}}, \mathrm{M}_{1}^{\beta \mathrm{ve}}, \mathrm{ABC}^{\mathrm{ve}}, \mathrm{M}_{2}^{\mathrm{ve}}, \mathrm{GA}^{\mathrm{ve}}, \mathrm{H}^{\mathrm{ve}}, \chi^{\mathrm{ve}}$, and $\mathrm{R}^{\mathrm{ve}}$ indexes for polymeric molecular network composed by Sierpinski networks $S(n, m)$, we compute the ev- and vedegree of each edge and vertex, respectively. Moreover, for mathematical estimation, calculations, and confirmation, MATLAB software was used. To plot these mathematical results, we used the software maple.

\section{Main Results}

This section deals with the computation of ve- and ev-degree-based topological indices for Sierpinski network $S(n, m)$. Let $m, n \geq 1$, then the Sierpinski network denoted by $S(n, m)$ is a graph on vertex set $V(S(n, m))=\{1,2, \ldots, m\}^{n}$. Any vertex $u \in V(S(n, m))$ has the representation $u=\left(u_{1}, u_{2}, \ldots, u_{n}\right)$, where $u_{l} \in\{1,2, \ldots, m\} \quad$ and $l \in 1,2, \ldots, n$. Two vertices $u, v \in V(S(n, m))$ are connected by an edge if and only if there exist and $p \in 1,2, \ldots, n$ such that
(i) $u_{q}=v_{q}$ for $q=1,2, \ldots, p-1$.
(ii) $u_{p}=v_{p}$, and
(iii) $u_{q}=v_{p}$ and $u_{p}=v_{q}$, for $p=q+1, \ldots, n$.

Sierpinski networks $S(n, m)$ definition comes from the study of Lipscomb's space. For $n=3$ in the definition of $S(n, m)$, we get the tower of Hanoi graph. The graphs of $S(2,5)$ and $S(3,5)$ are depicted in Figure 1. It is easy to

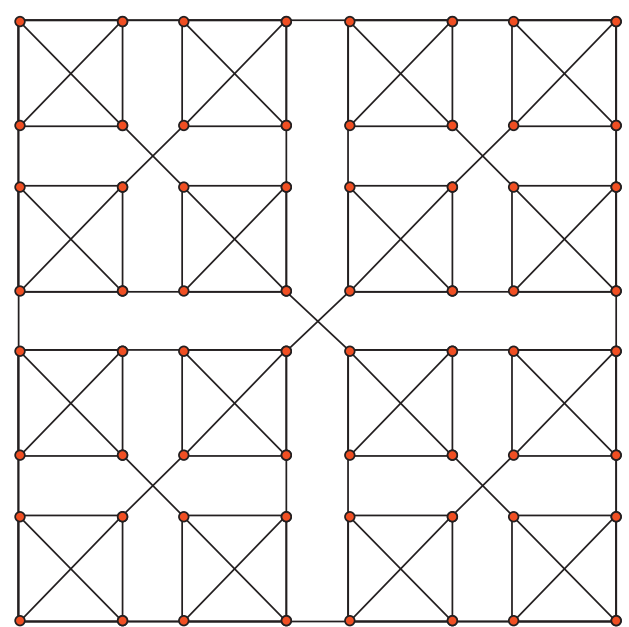

(a)

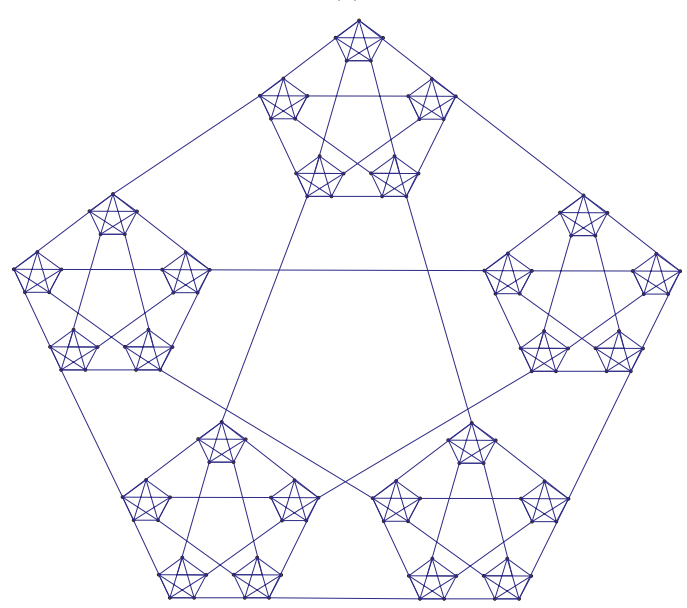

(b)

Figure 1: (a) The Sierpinski network S $(2,5)$. (b) The Sierpinski network $S(3,5)$.

compute that the number of vertices and number of edges of $S(n, m)$ are $m^{n}$ and $m^{n+1}-m / 2$, respectively.

Theorem 1. Let $\mathscr{H}$ be a graph of Sierpinski network $S(n, m)$, then

$$
\begin{aligned}
& \mathrm{M}^{\mathrm{ev}}(\mathscr{H})=\frac{1}{2}\left(7 m^{n+2}-4 m^{n}-7 m^{3}-2 m^{2}+6 m\right), \\
& \mathrm{R}^{\mathrm{ev}}(\mathscr{H})=\frac{m^{2}-m}{\sqrt{m+1}}+\frac{m^{n}-m}{2 \sqrt{2 m}}+\frac{m^{n+1}-m^{n}-2 m^{2}+2 m}{2 \sqrt{m+2}} .
\end{aligned}
$$

Proof. Let $E_{(p, q)}$ denote the set of edges of $\mathscr{H}$ with end vertices of degree $p$ and $q$, respectively. Then, $E(\mathscr{H})$ can be partitioned into two sets $E_{(m-1, m)}(\mathscr{H})$ and $E_{(m, m)}(\mathscr{H})$. The ev-degree of each edge in the partition sets is presented in Table 1.

From Table 1, the ev-degree-based Randic and Zagreb indices can be computed as 
TABle 1: ev-degree of each edge of $\mathscr{H}$.

\begin{tabular}{lcc}
\hline$\left(\Lambda\left(u_{1}\right), \Lambda\left(v_{1}\right)\right)$ & $\Lambda_{\mathrm{ev}}\left(e_{1}\right)$ & Frequency \\
\hline$E_{(m, m-1)}$ & $m+1$ & $m(m-1)$ \\
$E_{(m, m)}$ & $2 m$ & $\left(m^{n}-m\right) / 2$ \\
$E_{(m, m)}$ & $m+2$ & $m^{n+1}-m^{n}-2 m^{2}+2 m / 2$ \\
\hline
\end{tabular}

$$
\begin{aligned}
& \mathrm{M}^{\mathrm{ev}}(\mathscr{H})=\sum_{e_{1} \in E(\mathscr{H})} \Lambda_{\mathrm{ev}}\left(e_{1}\right)^{2} \\
& \mathrm{M}^{\mathrm{ev}}(\mathscr{H})=(m+1)^{2}\left|E_{(m, m-1)}\right|+(2 m)^{2}\left|E_{(m, m)}\right|+(m+2)^{2}\left|E_{(m, m)}\right| \\
& =(m+1)^{2}\left(m^{2}-m\right)+(2 m)^{2}\left(\frac{m^{n}-m}{2}\right)+(m+2)^{2}\left(\frac{m^{n+1}-m^{n}-2 m^{2}+2 m}{2}\right) \\
& =\frac{1}{2}\left(7 m^{n+2}-4 m^{n}-7 m^{3}-2 m^{2}+6 m\right), \\
& R^{\mathrm{ev}}(\mathscr{H})=\sum_{e_{1} \in E(\mathscr{H})} \Lambda_{\mathrm{ev}}\left(e_{1}\right)^{-(1 / 2)} \\
& R^{\mathrm{ev}}(\mathscr{H})=(m+1)^{-(1 / 2)}\left|E_{(m, m-1)}\right|+(2 m)^{-(1 / 2)}\left|E_{(m, m)}\right|+(m+2)^{-(1 / 2)}\left|E_{(m, m)}\right| \\
& =(m+1)^{-(1 / 2)}\left(m^{2}-m\right)+\frac{(2 m)^{-(1 / 2)}\left(m^{n}-m\right)}{2} \\
& +\frac{(m+2)^{-(1 / 2)}\left(m^{n+1}-m^{n}-2 m^{2}+2 m\right)}{2} \\
& =\frac{m^{2}-m}{\sqrt{m+1}}+\frac{m^{n}-m}{2 \sqrt{2 m}}+\frac{m^{n+1}-m^{n}-2 m^{2}+2 m}{2 \sqrt{m+2}}
\end{aligned}
$$

Theorem 2. Let $\mathscr{H}$ be a graph of Sierpinski network $S(n, m)$,

then

$$
\mathrm{M}_{1}^{\alpha \mathrm{ve}}(\mathscr{H})=\frac{1}{4}\left(m^{n+4}+6 m^{n+3}+5 m^{n+2}-12 m^{n+1}+4 m^{n}-8 m^{4}-16 m^{3}+32 m^{2}-12 m\right)
$$

Proof. Let $v_{1} \in V(\mathscr{H})$, then the degree of $v_{1}$ is either $m-1$ or $m$. The ve-degree of a vertex is either $m^{2}+m-2 / 2$ or $m^{2}+3 m-4 / 2$. These data are presented in Table 2. Using Table 2 , the value of $\mathrm{M}_{1}^{\alpha \mathrm{ve}}(\mathscr{H})$ can be calculated as

$$
\begin{aligned}
\mathrm{M}_{1}^{\alpha \mathrm{ve}}(\mathscr{H}) & =\sum_{v_{1} \in V(\mathscr{H})} \Lambda_{\mathrm{ve}}\left(v_{1}\right)^{2}, \\
\mathrm{M}_{1}^{\alpha \mathrm{ve}}(\mathscr{H}) & =\left(\frac{m^{2}+m-2}{2}\right)^{2}(m)+\left(\frac{m^{2}+3 m-4}{2}\right)^{2}\left(m^{2}-m\right)+\left(\frac{m^{2}+3 m-4}{2}\right)^{2}\left(m^{n}-m\right) \\
& =\frac{1}{4}\left(m^{n+4}+6 m^{n+3}+5 m^{n+2}-12 m^{n+1}+4 m^{n}-8 m^{4}-16 m^{3}+32 m^{2}-12 m\right) .
\end{aligned}
$$


TABle 2: ve-degree of each vertex of $\mathscr{H}$.

\begin{tabular}{lcc}
\hline$\Lambda\left(u_{1}\right)$ & $\Lambda_{\mathrm{ve}}\left(u_{1}\right)$ & Frequency \\
\hline$m-1$ & $m^{2}+m-2 / 2$ & $m$ \\
$m$ & $m^{2}+3 m-4 / 2$ & $m^{2}-m^{2}$ \\
$m$ & $m^{2}+3 m-2 / 2$ & $m^{n}-m^{2}$ \\
\hline
\end{tabular}

Theorem 3. Let $\mathscr{H}$ be a molecular graph of Sierpinski network $S(n, m)$, then

$$
\begin{aligned}
& \mathrm{M}_{1}^{\beta \mathrm{ve}}(\mathscr{H})=\frac{1}{4}\left(2 m^{n+3}+6 m^{n+2}-4 m^{n+1}-10 m^{3}+2 m^{2}+4 m\right), \\
& \mathrm{M}_{2}^{\mathrm{ve}}(\mathscr{H})=\frac{1}{8}\left(m^{n+5}+6 m^{n+4}+5 m^{n+3}-12 m^{n+2}+4 m^{n+1}-9 m^{5}-22 m^{4}+47 m^{3}-24 m^{2}+4 m\right), \\
& \operatorname{ABC}^{\mathrm{ve}}(\mathscr{H})=2\left(m^{2}-m\right)\left(\sqrt{\frac{m^{2}+2 m-5}{m^{4}+4 m^{3}-3 m^{2}-10 m+8}}\right)+\sqrt{\frac{m^{2}+3 m-5}{m^{4}+6 m^{3}+3 m^{2}-18 m}} \\
& +\left(m^{3}-3 m^{2}+2 m\right) \sqrt{\frac{m^{2}+3 m-6}{m^{4}+6 m^{3}+m^{2}-24 m+16}}+\frac{1}{4}\left(m^{n+1}-m^{3}-m^{2}+m\right) \sqrt{\frac{m^{2}+3 m-4}{m^{4}+5 m^{2}+6 m^{3}-12 m+4}} \\
& \mathrm{GA}^{\mathrm{ve}}(\mathscr{H})=\left(m^{2}-m\right)\left(\frac{\sqrt{m^{4}+4 m^{3}-3 m^{2}-10 m+8}}{m^{2}+2 m-3}+\frac{\sqrt{m^{4}+6 m^{3}+3 m^{2}-18 m}}{m^{2}+3 m-3}\right) \\
& +\frac{1}{2}\left[\left(m^{3}-3 m^{2}+2 m\right) \frac{\sqrt{m^{4}+6 m^{3}+m^{2}-24 m+16}}{m^{2}+3 m-4}+\left(m^{n+1}-m^{3}-m^{2}+m\right) \frac{\sqrt{m^{4}+5 m^{2}+6 m^{3}-12 m+4}}{m^{2}+3 m-2}\right], \\
& \mathrm{H}^{\mathrm{ve}}(\mathscr{H})=\frac{2 m}{m+3}+\frac{2\left(m^{2}-m\right)}{m^{2}+3 m-3}+\frac{m^{2}-2 m}{m+4}+\frac{m\left(m^{n}-m^{2}-m+1\right)}{m^{2}+3 m-2}, \\
& \chi^{\text {ve }}(\mathscr{H})=\left(m^{2}-m\right)\left(\frac{1}{\sqrt{m^{2}+2 m-3}}+\frac{1}{\sqrt{m^{2}+3 m-3}}\right)+\frac{1}{2}\left(\frac{m^{3}-3 m^{2}+2 m}{\sqrt{m^{2}+3 m-4}}+\frac{m^{n+1}-m^{3}-m^{2}+m}{\sqrt{m^{2}+3 m-2}}\right) \\
& \mathrm{R}^{\mathrm{ve}}(\mathscr{H})=2\left(m^{2}-m\right)\left(\frac{1}{\sqrt{m^{4}+4 m^{3}-3 m^{2}-10 m+8}}+\frac{1}{\sqrt{m^{4}+6 m^{3}+3 m^{2}-18 m+8}}\right) \\
& +\frac{m^{3}-3 m^{2}+2 m}{\sqrt{m^{4}+6 m^{3}+m^{2}-24 m+16}}+\frac{m^{n+1}-m^{3}-m^{2}+m}{\sqrt{m^{4}+6 m^{3}+5 m^{2}-12 m+4}}
\end{aligned}
$$

Proof. Based on the degree of end vertices of each edge, we can partition $E(\mathscr{H})$ into two sets $E_{(m-1, m)}$ and $E_{(m, m)}$. Now, depending on the ve-degree of end vertex of each edge, we can further partition the sets $E_{(m-1, m)}$ and $E_{(m, m)}$ into four sets. Table 3 shows the details of this partition. 
TABle 3: ev-degree of each edge of $\mathscr{H}$.

\begin{tabular}{lcc}
\hline Edge & $\left(\Lambda_{\mathrm{ve}}\left(u_{1}\right), \Lambda_{\mathrm{ve}}\left(v_{1}\right)\right)$ & Frequency \\
\hline$E_{(m-1, m)}^{*}$ & $\left(m^{2}+m-2 / 2, m^{2}+3 m-4 / 2\right)$ & $m^{2}-m^{2}$ \\
$E_{(m, m)}^{*}$ & $\left(\left(m^{2}+3 m-4\right) / 2,\left(m^{2}+3 m-2\right) / 2\right)$ & $m^{2}-m^{2}$ \\
$E_{(m, m)}^{*}$ & $\left(\left(m^{2}+3 m-4\right) / 2,\left(m^{2}+3 m-4\right) / 2\right)$ & $1 / 2\left(m^{3}-3 m^{2}+2 m\right)$ \\
$E_{(m, m)}^{*}$ & $\left(\left(m^{2}+3 m-2\right) / 2,\left(m^{2}+3 m-2\right) / 2\right)$ & $1 / 2\left(m^{n+1}-m^{3}-m^{2}+m\right)$ \\
\hline
\end{tabular}

Using Table 3, we can compute the indices as

$$
\begin{aligned}
& \mathrm{M}_{1}^{\beta \mathrm{ve}}(\mathscr{H})=\sum_{u_{1} v_{1} \in E(\mathscr{H})}\left(\Lambda_{\mathrm{ve}}\left(u_{1}\right)+\Lambda_{\mathrm{ve}}\left(v_{1}\right)\right) \\
& \mathrm{M}_{1}^{\beta \mathrm{ve}}(\mathscr{H})=\left(\frac{m^{2}+m-2}{2}+\frac{m^{2}+3 m-4}{2}\right)\left|E_{(m-1, m)}^{*}\right|+\left(\frac{m^{2}+3 m-4}{2}+\frac{m^{2}+3 m-2}{2}\right)\left|E_{(m-1, m)}^{*}\right| \\
& +\left(\frac{m^{2}+3 m-4}{2}+\frac{m^{2}+3 m-4}{2}\right)\left|E_{(m-1, m)}^{*}\right| \\
& +\left(\frac{m^{2}+3 m-2}{2}+\frac{m^{2}+3 m-2}{2}\right)\left|E_{(m-1, m)}^{*}\right| \\
& =\left(m^{2}+2 m-3\right)\left(m^{2}-m\right)+\left(m^{2}+3 m-3\right)\left(m^{2}-m\right)+\frac{1}{2}\left(m^{2}+3 m-4\right)\left(m^{3}-3 m^{2}+2 m\right) \\
& +\frac{1}{2}\left(m^{2}+3 m-2\right)\left(m^{n+1}-m^{3}-m^{2}+m\right) \\
& =\frac{1}{4}\left(2 m^{n+3}+6 m^{n+2}-4 m^{n+1}-10 m^{3}+2 m^{2}+4 m\right) \text {, } \\
& \mathrm{M}_{2}^{\mathrm{ve}}(\mathscr{H})=\sum_{u_{1} v_{1} \in E(\mathscr{H})}\left(\Lambda_{\mathrm{ve}}\left(u_{1}\right) \Lambda_{\mathrm{ve}}\left(v_{1}\right)\right) \\
& \mathrm{M}_{2}^{\mathrm{ve}}(\mathscr{H})=\left(\frac{m^{2}+m-2}{2}\right)\left(\frac{m^{2}+3 m-4}{2}\right)\left|E_{(m-1, m)}^{*}\right|+\left(\frac{m^{2}+3 m-4}{2}\right)\left(\frac{m^{2}+3 m-2}{2}\right)\left|E_{(m-1, m)}^{*}\right| \\
& +\left(\frac{m^{2}+3 m-4}{2}\right)\left(\frac{m^{2}+3 m-4}{2}\right)\left|E_{(m-1, m)}^{*}\right|+\left(\frac{m^{2}+3 m-2}{2}\right)\left(\frac{m^{2}+3 m-2}{2}\right)\left|E_{(m-1, m)}^{*}\right| \\
& =\frac{1}{4}\left(m^{4}+4 m^{3}-3 m^{2}-10 m+8\right)\left(m^{2}-m\right)+\frac{1}{4}\left(m^{2}-m\right)\left(m^{4}+6 m^{3}+3 m^{2}-18 m+8\right) \\
& +\frac{1}{8}\left(m^{4}+6 m^{3}+m^{2}-24 m+16\right)\left(m^{3}-3 m^{2}+2 m\right) \\
& +\frac{1}{8}\left(m^{4}+6 m^{3}+5 m^{2}-12 m+4\right)\left(m^{n+1}-m^{3}-m^{2}+m\right) \\
& =\frac{1}{8}\left(m^{n+5}+6 m^{n+4}+5 m^{n+3}-12 m^{n+2}+4 m^{n+1}-9 m^{5}-22 m^{4}+47 m^{3}-24 m^{2}+4 m\right) .
\end{aligned}
$$

\section{Numerical Discussions and Results}

In this portion, we have represented the numerical outcomes of the above computed topological descriptors related to the evdegrees and ve-degrees for molecular polymeric networks composed by Sierpinski network. We have computed numerical tables/results by using different values of $n$ and $k$ (see Tables 4, 5, and 6). Moreover, we have drawn the graphical results (see Figures 2-7) to test and review the behaviour of topological descriptors computed above. It is clear from Tables 4, 5, and 6 and Figures 2-Figure 7 that, as there is increase in $n$ and $k$, the computed topological descriptors are increasing. 
TABLe 4: Numerical table related to ev-degree-based indices.

\begin{tabular}{lcccccc}
\hline$[n, k]$ & {$[1,1]$} & {$[2,2]$} & {$[3,3]$} & {$[4,4]$} & {$[5,5]$} & {$[6,6]$} \\
\hline $\mathrm{M}^{\mathrm{ev}}$ & 0 & 22 & 702 & 13596 & $2.6674 \mathrm{e}+05$ & $5.7846 \mathrm{e}+06$ \\
$\mathrm{R}^{\mathrm{ev}}$ & 0 & 1.6547 & 17.29 & 201.78 & 2856.2 & 14282 \\
\hline
\end{tabular}

TABle 5: Numerical table related to ve-degree index.

\begin{tabular}{lccccccc}
\hline$[n, m]$ & {$[1,1]$} & {$[2,2]$} & {$[3,3]$} & {$[4,4]$} & {$[5,5]$} & {$[6,6]$} & {$[7,7]$} \\
\hline $\mathrm{M}_{1}^{\alpha \nu e}$ & 0 & 26 & 1521 & 42612 & 1126560 & 31536270 & 952009905 \\
\hline
\end{tabular}

TAвLE 6: Numerical table related to end vertices ve-degrees indices.

\begin{tabular}{lcccccc}
\hline$[n, m]$ & {$[1,1]$} & {$[2,2]$} & {$[3,3]$} & {$[4,4]$} & {$[5,5]$} & {$[6,6]$} \\
\hline $\mathrm{M}_{1}^{\beta \mathrm{ve}}$ & 0 & 16 & 588 & 13164 & 8277820 \\
$\mathrm{M}_{2}^{\mathrm{ve}}$ & 0 & 20 & 2229 & 55002 & $2.8157 \mathrm{e}+06$ & $9.4607 \mathrm{e}+07$ \\
$\mathrm{ABC}^{\mathrm{ve}}$ & 0 & 0.99654 & 5.4946 & 39.799 & 513.58 & 8978.6 \\
$\mathrm{GA}^{\mathrm{ve}}$ & 1403774.74 & 1403778.72 & 1403786.68 & 1403798.63 & 1403814.55 & 1403834.46 \\
$\mathrm{H}^{\mathrm{ve}}$ & 0.3 & 1.1214 & 5.2286 & 39.564 & 511.37 & 15097 \\
$\chi^{\mathrm{ve}}$ & 14917 & 14917 & 14928 & 14976 & 411.38 \\
$\mathrm{R}^{\mathrm{ve}}$ & 0 & 1.1438 & 5.2445 & 39.577 & 5383.6 \\
\hline
\end{tabular}

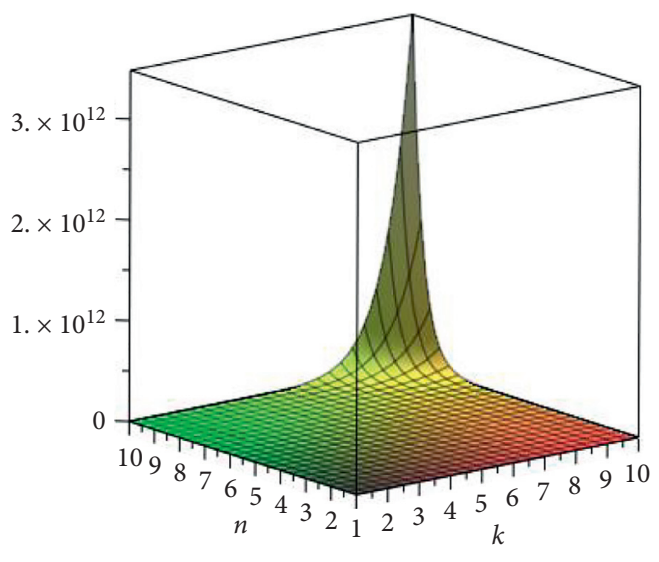

(a)

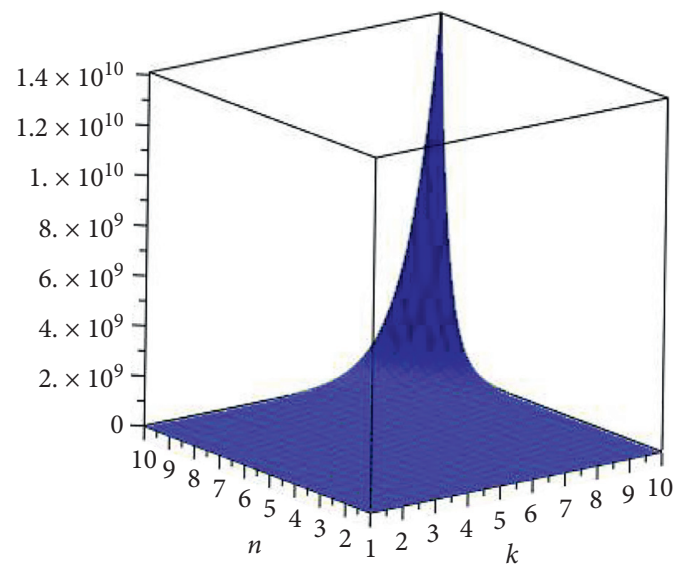

(b)

Figure 2: (a) The graph $\mathrm{M}^{\mathrm{ev}}(\mathscr{H})$. (b) The graph of $\mathrm{R}^{\mathrm{ev}}(\mathscr{H})$. 


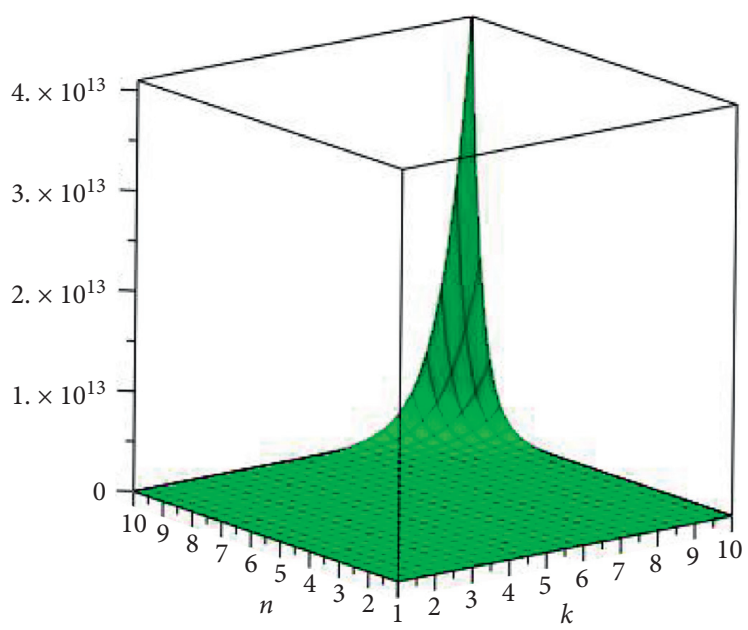

Figure 3: The graph of $\mathrm{M}_{1}^{\alpha v e}(\mathscr{H})$.

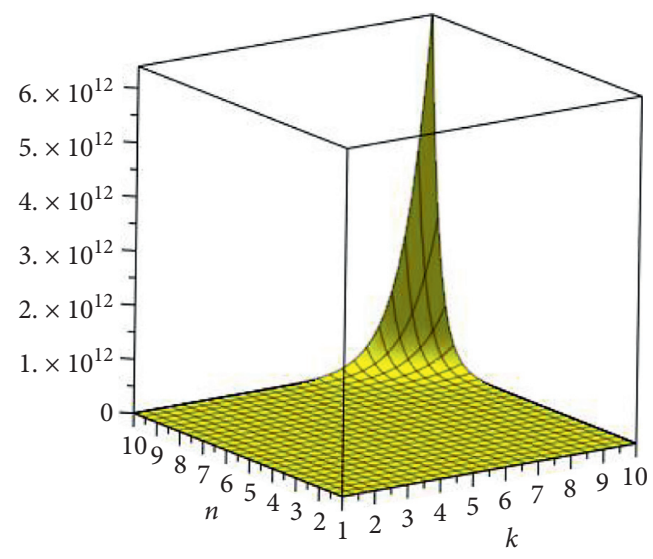

(a)

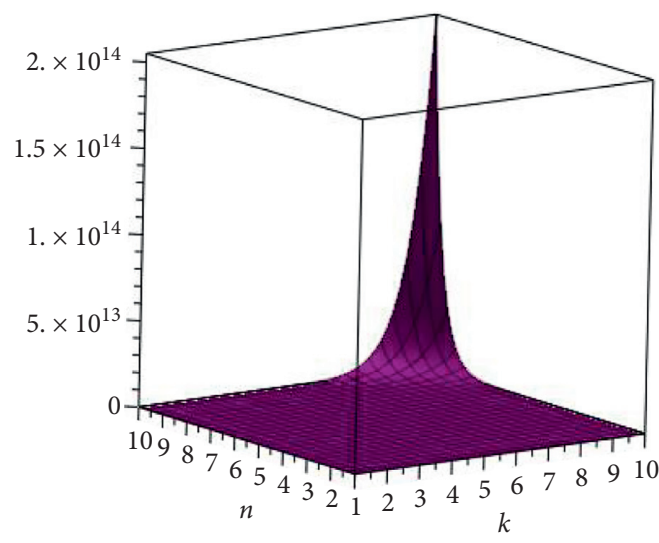

(b)

Figure 4: (a) The graph of $\mathrm{M}_{1}^{\beta \mathrm{ve}}(\mathscr{H})$. (b) The graph of $\mathrm{M}_{2}^{\mathrm{ve}}(\mathscr{H})$.

$$
\begin{aligned}
\operatorname{ABC}^{\mathrm{ve}}(\mathscr{H})= & \sum_{u_{1} v_{1} \in E(\mathscr{H})} \sqrt{\frac{\Lambda_{\mathrm{ve}}\left(u_{1}\right)+\Lambda_{\mathrm{ve}}\left(v_{1}\right)-2}{\left(\Lambda_{\mathrm{ve}}\left(u_{1}\right) \Lambda_{\mathrm{ve}}\left(v_{1}\right)\right)}} \\
\operatorname{ABC}^{\mathrm{ve}}(\mathscr{H})= & 2 \sqrt{\frac{m^{2}+2 m-5}{m^{4}+4 m^{3}-3 m^{2}-10 m+8}}\left|E_{(m-1, m)}^{*}\right| \\
& +2 \sqrt{\frac{m^{2}+3 m-5}{m^{4}+6 m^{3}+3 m^{2}-18 m}}\left|E_{(m-1, m)}^{*}\right| \\
& +2 \sqrt{\frac{m^{2}+3 m-6}{m^{4}+6 m^{3}+m^{2}-24 m+16}}\left|E_{(m-1, m)}^{*}\right|
\end{aligned}
$$




$$
\begin{aligned}
& +2 \sqrt{\frac{m^{2}+3 m-4}{m^{4}+5 m^{2}+6 m^{3}-12 m+4}}\left|E_{(m-1, m)}^{*}\right| \\
& =2\left(m^{2}-m\right) \sqrt{\frac{m^{2}+2 m-5}{m^{4}+4 m^{3}-3 m^{2}-10 m+8}}+2\left(m^{2}-m\right) \sqrt{\frac{m^{2}+3 m-5}{m^{4}+6 m^{3}+3 m^{2}-18 m}} \\
& +\left(m^{3}-3 m^{2}+2 m\right) \sqrt{\frac{m^{2}+3 m-6}{m^{4}+6 m^{3}+m^{2}-24 m+16}} \\
& +\frac{1}{4}\left(m^{n+1}-m^{3}-m^{2}+m\right) \sqrt{\frac{m^{2}+3 m-4}{m^{4}+5 m^{2}+6 m^{3}-12 m+4}} \\
& =2\left(m^{2}-m\right)\left(\sqrt{\frac{m^{2}+2 m-5}{m^{4}+4 m^{3}-3 m^{2}-10 m+8}}+\sqrt{\frac{m^{2}+3 m-5}{m^{4}+6 m^{3}+3 m^{2}-18 m}}\right) \\
& +\left(m^{3}-3 m^{2}+2 m\right) \sqrt{\frac{m^{2}+3 m-6}{m^{4}+6 m^{3}+m^{2}-24 m+16}} \\
& +\frac{1}{4}\left(m^{n+1}-m^{3}-m^{2}+m\right) \sqrt{\frac{m^{2}+3 m-4}{m^{4}+5 m^{2}+6 m^{3}-12 m+4}}, \\
& \mathrm{GA}^{\mathrm{ve}}(\mathscr{H})=\sum_{u_{1} v_{1} \in E(\mathscr{H})} \frac{2 \sqrt{\Lambda_{\mathrm{ve}}\left(u_{1}\right) \times \Lambda_{\mathrm{ve}}\left(v_{1}\right)}}{\left(\Lambda_{\mathrm{ve}}\left(u_{1}\right)+\Lambda_{\mathrm{ve}}\left(v_{1}\right)\right)}, \\
& \mathrm{GA}^{\mathrm{ve}}(\mathscr{H})=\frac{\sqrt{m^{4}+4 m^{3}-3 m^{2}-10 m+8}}{m^{2}+2 m-3}\left|E_{(m-1, m)}^{*}\right|+\frac{\sqrt{m^{4}+6 m^{3}+3 m^{2}-18 m}}{m^{2}+3 m-3}\left|E_{(m-1, m)}^{*}\right| \\
& +\frac{\sqrt{m^{4}+6 m^{3}+m^{2}-24 m+16}}{2\left(m^{2}+3 m-4\right)}\left|E_{(m-1, m)}^{*}\right|+\frac{\sqrt{m^{4}+6 m^{3}+5 m^{2}-12 m+4}}{2\left(m^{2}+3 m-2\right)}\left|E_{(m-1, m)}^{*}\right| \\
& =\left(m^{2}-m\right) \frac{\sqrt{m^{4}+4 m^{3}-3 m^{2}-10 m+8}}{m^{2}+2 m-3}+\left(m^{2}-m\right) \frac{\sqrt{m^{4}+6 m^{3}+3 m^{2}-18 m}}{m^{2}+3 m-3} \\
& +\left(m^{3}-3 m^{2}+2 m\right) \frac{\sqrt{m^{4}+6 m^{3}+m^{2}-24 m+16}}{2\left(m^{2}+3 m-4\right)} \\
& +\left(m^{n+1}-m^{3}-m^{2}+m\right) \frac{\sqrt{m^{4}+6 m^{3}+5 m^{2}-12 m+4}}{2\left(m^{2}+3 m-2\right)} \\
& =\left(m^{2}-m\right)\left(\frac{\sqrt{m^{4}+4 m^{3}-3 m^{2}-10 m+8}}{m^{2}+2 m-3}+\frac{\sqrt{m^{4}+6 m^{3}+3 m^{2}-18 m}}{m^{2}+3 m-3}\right) \\
& +\left(m^{3}-3 m^{2}+2 m\right) \frac{\sqrt{m^{4}+6 m^{3}+m^{2}-24 m+16}}{2\left(m^{2}+3 m-4\right)} \\
& +\left(m^{n+1}-m^{3}-m^{2}+m\right) \frac{\sqrt{m^{4}+6 m^{3}+5 m^{2}-12 m+4}}{2\left(m^{2}+3 m-2\right)} \text {. }
\end{aligned}
$$


10

Mathematical Problems in Engineering

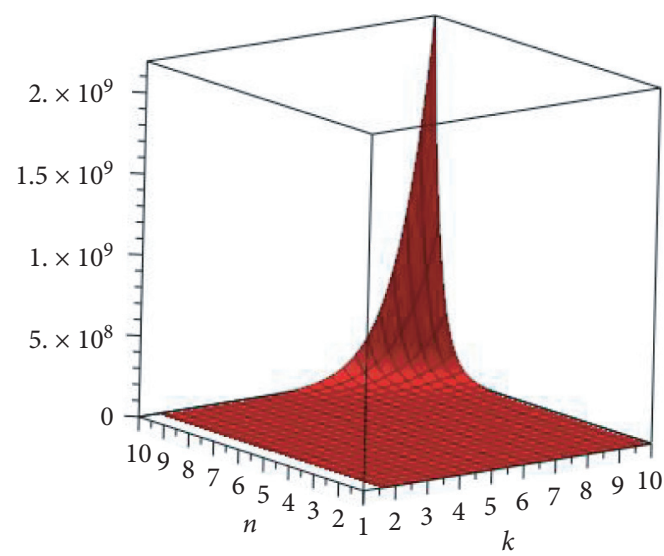

(a)

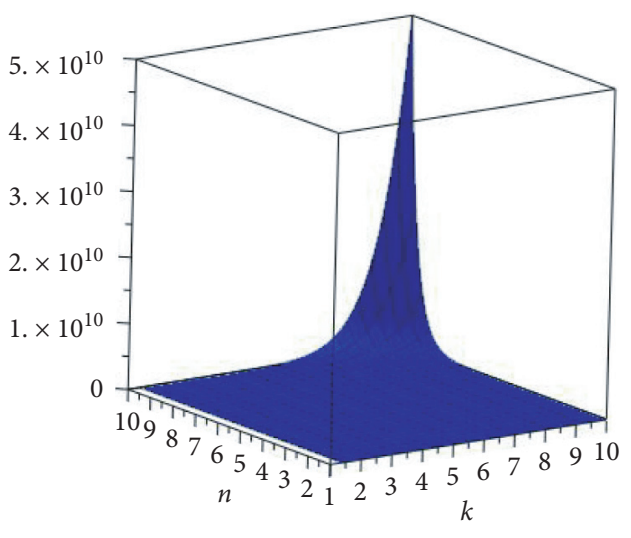

(b)

Figure 5: (a) The graph of $\operatorname{ABC}^{\mathrm{ve}}(\mathscr{H})$. (b) The graph of $\mathrm{GA}^{\mathrm{ve}}(\mathscr{H})$.

$$
\begin{aligned}
& \mathrm{H}^{\mathrm{ve}}(\mathscr{H})=\sum_{u_{1} v_{1} \in E(\mathscr{H})} \frac{2}{\Lambda_{\mathrm{ve}}\left(u_{1}\right)+\Lambda_{\mathrm{ve}}\left(v_{1}\right)} \\
& \mathrm{H}^{\mathrm{ve}}(\mathscr{H})=\left(\frac{2}{m^{2}+2 m-3}\right)\left|E_{(m-1, m)}^{*}\right|+\left(\frac{2}{m^{2}+3 m-3}\right)\left|E_{(m, m)}^{*}\right|+\left(\frac{1}{m^{2}+3 m-4}\right)\left|E_{(m, m)}^{*}\right| \\
& +\left(\frac{1}{m^{2}+3 m-2}\right)\left|E_{(m, m)}^{*}\right| \\
& =\left(\frac{2}{m^{2}+2 m-3}\right)\left(m^{2}-m\right)+\left(\frac{2}{m^{2}+3 m-3}\right)\left(m^{2}-m\right) \\
& +\left(\frac{1}{2 m^{2}+3 m-4}\right)\left(m^{3}-3 m^{2}+2 m\right)+\left(\frac{1}{m^{2}+3 m-2}\right)\left(m^{n+1}-m^{3}-m^{2}+m\right) \\
& =\frac{2 m}{m+3}+\frac{2 m(m-1)}{m^{2}+3 m-3}+\frac{m(m-2)}{m+4}+\frac{m\left(m^{n}-m^{2}-m+1\right)}{m^{2}+3 m-2}, \\
& \chi^{\mathrm{ve}}(\mathscr{H})=\sum_{u_{1} v_{1} \in E(\mathscr{H})}\left(\Lambda_{\mathrm{ve}}\left(u_{1}\right)+\Lambda_{\mathrm{ve}}\left(v_{1}\right)\right)^{-(1 / 2)} \\
& =\chi^{\mathrm{ve}}(\mathscr{H})=\left(m^{2}+2 m-3\right)^{-(1 / 2)}\left|E_{(m-1, m)}^{*}\right|+\left(m^{2}+3 m-3\right)^{-(1 / 2)}\left|E_{(m-1, m)}^{*}\right|+\left(m^{2}+3 m-4\right)^{-(1 / 2)}\left|E_{(m-1, m)}^{*}\right| \\
& +\left(m^{2}+3 m-2\right)^{-(1 / 2)}\left|E_{(m-1, m)}^{*}\right| \\
& =\left(m^{2}+2 m-3\right)^{-(1 / 2)}\left(m^{2}-m\right)+\left(m^{2}+3 m-3\right)^{-(1 / 2)}\left(m^{2}-m\right) \\
& +\frac{1}{2}\left(m^{2}+3 m-4\right)^{-(1 / 2)}\left(m^{3}-3 m^{2}+2 m\right)+\frac{1}{2}\left(m^{2}+3 m-2\right)^{-(1 / 2)}\left(m^{n+1}-m^{3}-m^{2}+m\right) \\
& +\frac{1}{2}\left(\frac{m^{3}-3 m^{2}+2 m}{\sqrt{m^{2}+3 m-4}}+\frac{m^{n+1}-m^{3}-m^{2}+m}{\sqrt{m^{2}+3 m-2}}\right) .
\end{aligned}
$$




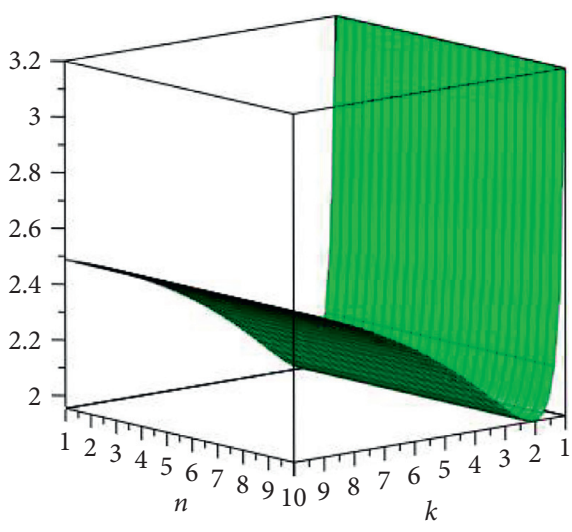

(a)

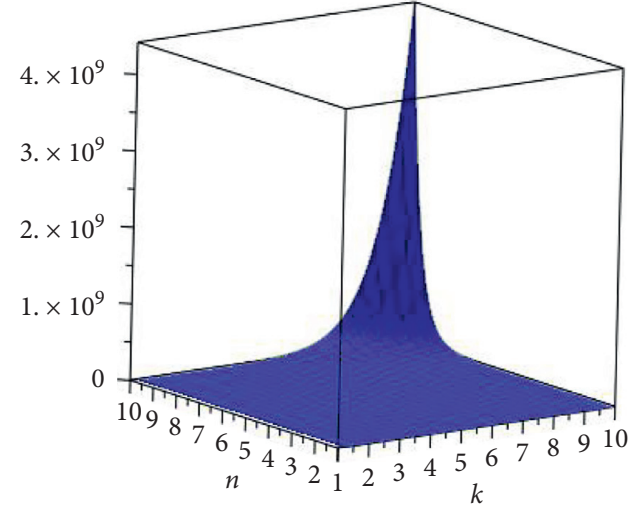

(b)

Figure 6: (a) The graph of $\mathrm{H}^{\mathrm{ve}}(\mathscr{H})$. (b) The graph of $\chi^{\mathrm{ve}}(\mathscr{H})$.

$$
\begin{aligned}
& \mathrm{R}^{\mathrm{ve}}(\mathscr{H})=\sum_{u_{1} v_{1} \in E(\mathscr{H})}\left(\Lambda_{\mathrm{ve}}\left(u_{1}\right) \Lambda_{\mathrm{ve}}\left(v_{1}\right)\right)^{-(1 / 2)}, \\
& \mathrm{R}^{\mathrm{ve}}(\mathscr{H})=\left[\frac{1}{4}\left(m^{4}+4 m^{3}-3 m^{2}-10 m+8\right)\right]^{-(1 / 2)}\left|E_{(m-1, m)}^{*}\right| \\
& +\left[\frac{1}{4}\left(m^{4}+6 m^{3}+3 m^{2}-18 m+8\right)\right]^{-(1 / 2)}\left|E_{(m-1, m)}^{*}\right| \\
& +\left[\frac{1}{4}\left(m^{4}+6 m^{3}+m^{2}-24 m+16\right)\right]^{-(1 / 2)}\left|E_{(m-1, m)}^{*}\right| \\
& +\left[\frac{1}{4}\left(m^{4}+6 m^{3}+5 m^{2}-12 m+4\right)\right]^{-(1 / 2)}\left|E_{(m-1, m)}^{*}\right| \\
& =\left[\frac{1}{4}\left(m^{4}+4 m^{3}-3 m^{2}-10 m+8\right)\right]^{-(1 / 2)}\left(m^{2}-m\right) \\
& +\left[\frac{1}{4}\left(m^{4}+6 m^{3}+3 m^{2}-18 m+8\right)\right]^{-(1 / 2)}\left(m^{2}-m\right) \\
& +\frac{1}{2}\left[\frac{1}{4}\left(m^{4}+6 m^{3}+m^{2}-24 m+16\right)\right]^{-(1 / 2)}\left(m^{3}-3 m^{2}+2 m\right) \\
& +\frac{1}{2}\left[\frac{1}{4}\left(m^{4}+6 m^{3}+5 m^{2}-12 m+4\right)\right]^{-(1 / 2)}\left(m^{n+1}-m^{3}-m^{2}+m\right) \\
& =\frac{2\left(m^{2}-m\right)}{\sqrt{m^{4}+4 m^{3}-3 m^{2}-10 m+8}}+\frac{2\left(m^{2}-m\right)}{\sqrt{m^{4}+6 m^{3}+3 m^{2}-18 m+8}} \\
& +\frac{m^{3}-3 m^{2}+2 m}{\sqrt{m^{4}+6 m^{3}+m^{2}-24 m+16}}+\frac{m^{n+1}-m^{3}-m^{2}+m}{\sqrt{m^{4}+6 m^{3}+5 m^{2}-12 m+4}} .
\end{aligned}
$$




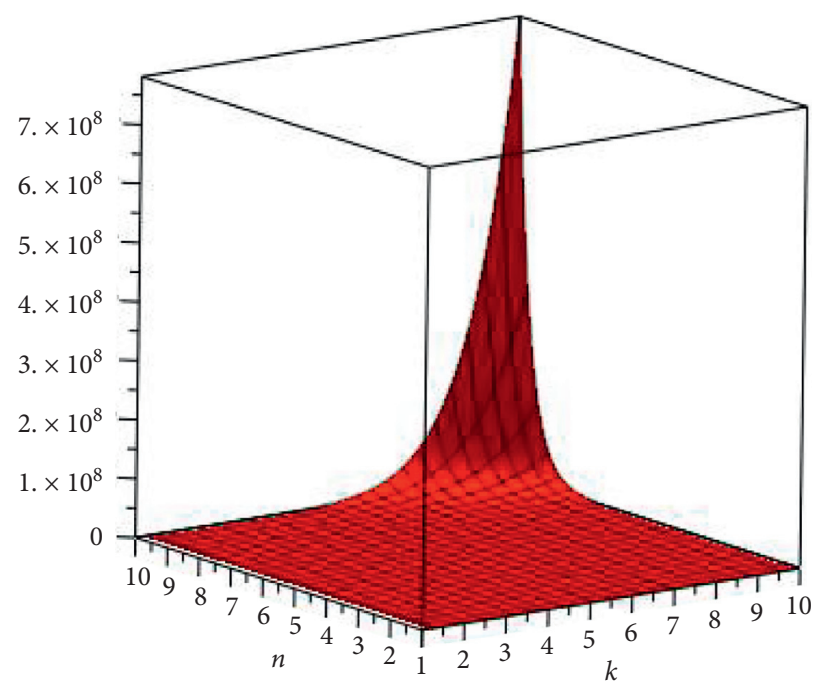

Figure 7: The graph of $\mathrm{R}^{\mathrm{ve}}(\mathscr{H})$.

\section{Conclusion}

To understand the basic topology, the analysis of chemical graphs and networks by topological descriptors is required. Such research has a broad variety of uses in the fields of bioinformatics, cheminformatics, biostatistics, and drug delivery, where a variety of daunting schemes are tackled by diagnostics based on different graph endeavours. Graphs are essential methods for estimating and forecasting the characteristics of chemical and biological compounds in the study of QSPR and QSAR.

In this paper, we have presented the results for ev- and ve-degree-based topological indices for the polymeric molecular networks modeled by Sierpinski networks that can be utilized for QSPR and QSAR study.

\section{Data Availability}

No data are required to support the study.

\section{Conflicts of Interest}

The authors declare that they have no conflicts of interest.

\section{References}

[1] R. Gozalbes, J. Doucet, and F. Derouin, “Application of topological descriptors in QSAR and drug design: history and new trends," Current Drug Targets-Infectious Disorders, vol. 2, no. 1, pp. 93-102, 2002.

[2] P. J. Hansen and P. C. Jurs, "Chemical applications of graph theory. Part I. Fundamentals and topological indices," Journal of Chemical Education, vol. 65, no. 7, p. 574, 1988.

[3] D. H. Rouvray and R. B. King, Topology in Chemistry: Discrete Mathematics of Molecules, Elsevier, Amsterdam, Netherlands, 2002.

[4] M. Imran, W. Sabeel-e-Hafi, W. Gao, and M. Reza Farahani, "On topological properties of sierpinski networks," Chaos, Solitons \& Fractals, vol. 98, pp. 199-204, 2017.
[5] M. Randic, "Characterization of molecular branching," Journal of the American Chemical Society, vol. 97, no. 23, pp. 6609-6615, 1975.

[6] M. Randic, Chemometrics and Intelligent Laboratory Systems, vol. 10, p. 213, 1991.

[7] A. T. Balaban, I. Motoc, D. Bonchev, O. Mekenyan, M. Charton, and I. Motoc, Eds., Topics in Current Chemistry, vol. 114p. 21, 1983.

[8] A. T. Balaban, "Topological indices and their uses: a new approach for the coding of alkanes," Journal of Molecular Structure: Theochem, vol. 165, no. 3-4, pp. 243-253, 1988.

[9] A. T. Balaban, "Chemical graphs," Theoretica Chimica Acta, vol. 53, no. 4, pp. 355-375, 1979.

[10] D. B. West, Introduction to Graph Theory, Prentice-Hall, New Jersey, USA, second edition, 2003.

[11] B. Bollobas and P. Erdos, "Distance irredundance in graphs: complexity issues," Ars Combinatoria, vol. 50, pp. 225-233, 1998.

[12] I. Gutman and N. Trinajstić, "Graph theory and molecular orbitals. Total $\varphi$-electron energy of alternant hydrocarbons," Chemical Physics Letters, vol. 17, no. 4, pp. 535-538, 1972.

[13] I. Gutman, B. Ruscic, N. Trinajstic, and C. F. Wilcox, "Graph theory and molecular orbitals. XII. Acyclic polyenes," The Journal of Chemical Physics, vol. 62, no. 9, pp. 3399-3405, 1975.

[14] B. Zhou and N. Trinajstić, "On a novel connectivity index," Journal of Mathematical Chemistry, vol. 46, no. 4, pp. 12521270, 2009.

[15] L. Zhong, "The harmonic index for graphs," Applied Mathematics Letters, vol. 25, no. 3, pp. 561-566, 2012.

[16] E. Estrada, L. Torres, L. Rodriguez, and I. Gutman, "News and announcements," Journal of Endodontics, vol. 24, no. 12, pp. 849-855, 1998.

[17] E. Estrada, "Generalization of topological indices," Chemical Physics Letters, vol. 336, no. 3-4, pp. 248-252, 2001.

[18] B. Vukičević and B. Furtula, "Topological index based on the ratios of geometrical and arithmetical means of end-vertex degrees of edges," Journal of Mathematical Chemistry, vol. 46, pp. 1369-1376, 2009.

[19] M. Chellali, T. W. Haynes, S. T. Hedetniemi, and T. M. Lewis, "On ve-degrees and ev-degrees in graphs," Discrete Mathematics, vol. 340, no. 2, pp. 31-38, 2017. 
[20] B. Horoldagva, K. C. Das, and T.-A. Selenge, "On ve-degree and ev-degree of graphs," Discrete Optimization, vol. 31, pp. 1-7, 2019.

[21] S. Ediz, "On ve-degree molecular topological properties of silicate and oxygen networks," International Journal of Computing Science and Mathematics, vol. 9, no. 1, pp. 1-12, 2018.

[22] M. Cancan, "On ev-degree and ve-degree topological properties of tickysim spiking neural network," Computational Intelligence and Neuroscience, vol. 2019, 2019.

[23] Z.-Q. Cai, A. Rauf, M. Ishtiaq, and M. K. Siddiqui, "On vedegree and ev-degree based topological properties of silicon carbide Si2C3-II [p, q]," Polycyclic Aromatic Compounds, vol. 75, pp. 1-15, 2020.

[24] X. Zhang, A. Rauf, M. Ishtiaq, M. K. Siddiqui, and M. H. Muhammad, "On degree based topological properties of two carbon nanotubes," Polycyclic Aromatic Compounds, vol. 107, pp. 1-19, 2020.

[25] Y.-M. Chu, M. K. Siddiqui, M. F. Hanif, A. Rauf, M. Ishtiaq, and M. H. Muhammad, "On ve-degree and ev-degree based topological properties of H-naphtalenic nanotube," Polycyclic Aromatic Compounds, vol. 12, pp. 1-13, 2020. 